\title{
Navigating the Labyrinth of Pharmacogenetic Testing: A Guide to Test Selection
}

\author{
Chad A Bousman ${ }^{1,2,3}$, Heather Zierhut ${ }^{4}$, Daniel J. Müller, MD PhD ${ }^{5,6}$
}

${ }^{1}$ Departments of Medical Genetics, Psychiatry, Physiology \& Pharmacology, University of Calgary, Calgary, $A B$, Canada

${ }^{2}$ Alberta Children's Hospital Research Institute, Calgary, AB, Canada

${ }^{3}$ Mathison Centre for Mental Health Research and Education, Hotchkiss Brain Institute, Cumming School of Medicine, University of Calgary, Calgary, AB, Canada

${ }^{4}$ Department of Genetics, Cell Biology, and Development, University of Minnesota, Minneapolis, MN, USA

${ }^{5}$ Pharmacogenetics Research Clinic, Campbell Family Mental Health Research Institute, Centre for Addiction and Mental Health, Toronto, ON, Canada

${ }^{6}$ Department of Psychiatry, University of Toronto, Toronto, ON, Canada

\section{Correspondence:}

Dr. Chad Bousman, chad.bousman@ucalagary.ca

Department of Medical Genetics, University of Calgary, 228 HMRB, 3330 Hospital Drive NW, Calgary, AB T2N 4N1, Canada

Tel: +1 (403) 441-8409

Key words: Pharmacogenomics; Decision-making tools; Resources; Guidance

\section{CONFLICT OF INTEREST}

C.A.B has received material support from Assurex Health (Myriad Neuroscience), CNSDose, Genomind, and AB-Biotics for research purposes and has ongoing research collaborations with CNSDose and MyDNA but does not have equity, stocks, or options in these companies or any This is the author manuscript accepted for publication and has undergone full peer review but has not been through the copyediting, typesetting, pagination and proofreading process, which may lead to differences between this version and the Version of Record. Please cite this article as doi: $\underline{10.1002 / C P T .1432}$

This article is protected by copyright. All rights reserved 
other pharmacogenetic companies. H.Z. is an advisory board member of GeneMatters, LLC, a telehealth genetic counseling company but does not have equity, stocks, or options in these companies or any other pharmacogenetic companies. D.J.M. is co-investigator in two pharmacogenetic studies where genetic test kits were provided as in-kind contribution by Assurex Health (Myriad Neuroscience) to evaluate feasibility of pharmacogenetic testing in clinical practice and potential benefits of pharmacogenetic testing compared to treatment as usual. D.J.M. has not received any payments or received any equity, stocks, or options from this company or any other pharmacogenetic companies. D.J.M. is co-investigator in two filed genetic patents assessing risk for antipsychotic-induced weight gain. C.A.B and D.J.M are members of Clinical Pharmacogenetics Implementation Consortium and the Genetic Testing Committee of the International Society of Psychiatric Genetics.

\section{FUNDING}

No funding was received for this work.

\section{INTRODUCTION}

Numerous commercial laboratories offer clinical pharmacogenetic testing in the US and abroad. With many pharmacogenetic testing choices, healthcare providers (e.g., pharmacists, physicians, genetic counsellors) are often tasked with deciding which test best suits the needs of their patients and clinical practice. Unfortunately, no guidelines exist on how to select a test with appropriate analytical and clinical validity. Here, we provide information and resources to assist providers in the identification and selection of appropriate pharmacogenetic tests.

\section{WELCOME TO THE LABYRINTH}

Despite the provision of pharmacogenetic testing in a growing number of medical centers and health systems, the past decade has witnessed an exponential growth in the number of commercial laboratories offering clinical pharmacogenetic testing. This has created a situation in which most healthcare providers, particularly in the US, have access to multiple pharmacogenetic testing options. Unfortunately, deciding which testing laboratory to use is often not straightforward and complicated by the fact that pharmacogenetic testing is largely unregulated and not standardized. As a result, there is significant variability between tests in regards to gene and allele content, test result interpretation, cost, and testing turnaround times. Despite knowledge of this variability, there is limited indication that regulation or

This article is protected by copyright. All rights reserved 
standardization of pharmacogenetic testing will be implemented in the near future, leaving healthcare providers with the arduous task of navigating through the current labyrinth of pharmacogenetic testing options. Overcoming this burden however, can be accomplished and suitable pharmacogenetic tests can be identified that match the needs of providers and their patients.

\section{PHARMACOGENETIC TEST SELECTION}

Herein, we discuss a series of topics and highlight resources that when considered and consulted, will increase the probability of selecting pharmacogenetic tests with acceptable analytical and clinical validity. We cannot however, make assurances that this discussion or the resources highlighted will result in the selection of a test with clinical utility, as this will largely depend on the clinical context in which the test is being implemented. For quick reference, we have created a decision tree (Figure 1) highlighting key questions and resources essential to the decision-making process, described in detail below.

\section{Actionable gene-drug pairs can be found through online databases}

A considerable proportion of drugs have pharmacogenetic-based dosing guidelines or labels with pharmacogenetic advice. The Pharmacogenomics Knowledgebase (PharmGKB, www.pharmgkb.org) lists 90 unique gene-drug pairs comprising 65 drugs and 21 genes with pharmacogenetic-based guidelines developed by the Clinical Pharmacogenetics Implementation Consortium (CPIC, www.cpicpgx.org), Royal Dutch Association for the Advancement of Pharmacy - Pharmacogenetics Working Group (DPWG, www.pharmgkb.org/page/dpwg), and the Canadian Pharmacogenomics Network for Drug Safety (CPNDS, www.cpnds.ubc.ca) (Figure 2). Drug labels approved by the US Food and Drug Administration, European Medicines Agency, Japan's Pharmaceutical and Medical Devices Agency, and Health Canada add a further 229 actionable gene-drug pairs comprising an additional 139 drugs and 66 genes. Notably, most of the implicated drugs are routinely prescribed and some are first-line therapeutic options, suggesting that in many cases ordering a pharmacogenetic test will be a reasonable option for informing dosing and selection of initial drug therapy and in some cases future drug therapy.

This article is protected by copyright. All rights reserved 


\section{Laboratories can be found through online registries and should be accredited}

At least 76 laboratories offer clinical pharmacogenetic testing services in the US ${ }^{1}$ and although other countries do not have as many choices, finding a laboratory that offers testing is typically not a challenge. The challenge lies in finding an accredited laboratory that offers testing for the specific gene-drug pair(s) relevant to a particular clinical situation. In the US, the NIH Genetic Testing Registry (www.ncbi.nlm.nih.gov/gtr/), McKesson Diagnostics Exchange (app.dexzcodes.com), and Association for Molecular Pathology's Test Directory (www.amp.org/resources/test-directory/) are resources for identification of accredited laboratories. However, registration of a test is optional and as such these resources are not inclusive of all laboratories offering pharmacogenetic testing. Whether or not one of these resources is utilized to find a laboratory, it is imperative that the selected laboratory's testing procedures are evaluated and accredited by an independent third-party (e.g., Clinical Laboratory Improvement Amendments, CLIA) to ensure a high level of analytical validity.

\section{Test gene and allele content should be evidence based}

Pharmacogenetic tests are not standardized. Depending on the laboratory, testing is offered as a single- or multiple-gene panel. From a clinical value perspective, multiple-gene panels are preferred as they provide more information and reduce the need for future testing, at a modest increase in cost. The task is in determining which test panel comprises the optimal set of genes and alleles.

Limiting a testing panel to genes with pharmacogenetic-based dosing guidelines allows for a high degree of clinical validity. However, consensus on the optimum set of alleles to test within these genes is largely absent. ${ }^{2,3}$ One issue leading to the complexity in proposing a set list of alleles is that differences in the combination of alleles included on a testing panel can lead to discordant drug selection and dosing advice. ${ }^{3}$ Thus, it is critical to evaluate the allele content of a test prior to ordering. To inform this evaluation the Pharmacogene Variant Consortium (PharmVar, www.pharmvar.org), a repository and authority for allele designation within clinically actionable genes, is a good starting point. PharmVar provides a list of known variant alleles (i.e., star alleles or haplotypes) for clinically actionable genes along with the function (i.e., normal, increased, decreased, no function) of each allele according to information 
curated by PharmGKB and CPIC. The more alleles a test includes with a functional impact on a gene, the more confidence one should have in the predicted phenotype and associated drug selection and dosing advice.

The patient's ethnic background is another important but often overlooked factor to consider when evaluating a test's allele content. The frequencies of actionable alleles can vary by ethnicity. For example, the CYP2D6*29 decreased function allele is rare in individuals of European background (0.1\%, range: $0 \%$ - 1\%) but common among those of African background $(9 \% \text {, range: } 4 \%-20 \%)^{4}$. Testing laboratories differ in their attention to this issue and test panels tend to be biased toward the inclusion of alleles most common in populations of European descent, which can result in an increase in false negatives for non-Europeans. Using the same example as above, a test panel that did not include the CYP2D6*29 allele would inadvertently assign some Africans as normal metabolizers instead of intermediate metabolizers. Thus, when selecting a test, particularly in an individual of non-European background, it is important to be aware of the alleles being tested. If a laboratory does not or will not provide a list of the alleles being tested, extreme caution should be taken or alternative laboratories explored.

\section{Test results and interpretation should be transparent}

The method by which genotyping results are translated into phenotypes (e.g., metabolizer status) and then used to generate drug selection and dosing recommendations continues to evolve. Many calls for standardization of this process have been made but differences between laboratories still remain. As such, understanding how a laboratory translates genotyping results into phenotypes and further into recommendations is important. Transparency is key but many laboratories use proprietary algorithms, which prevents full transparency. Nonetheless, all laboratories should provide the actual test results (i.e., results for each variant/SNP genotyped) and when applicable, non-genetic factors (e.g. smoking status) that are included in their algorithms and cite the evidence that underlies the clinical recommendations they offer. Resistance to provide this information should signal concerns about the quality of the results and recommendations as well as their applicability to clinical practice.

\section{Test feasibility should match the setting}

This article is protected by copyright. All rights reserved 
Some may contend that an evaluation of the feasibility (i.e., accessibility, affordability, and efficiency) of a pharmacogenetic test should precede the considerations discussed thus far. We would argue that issues of feasibility will largely be mitigated in the future as the number of laboratories offering tests continues to grow and the costs associated with testing steadily drops. Nonetheless, feasibility concerns play heavily into current decisions to order a test. As noted earlier, accessibility of pharmacogenetic testing, particularly in the US, has become a minor issue due to the critical mass of testing laboratories including direct-to-consumer testing companies (e.g., 23andMe). However, pharmacogenetic testing efficiency (i.e., testing turnaround times) and affordability remain unresolved. Turnaround times range from a few days to three weeks and are mainly a function of the methods used to collect and transport specimens and the efficiency of the technology used to perform the testing. Depending on the clinical context, turnaround time might be weighted more (e.g., crisis/acute care settings) or less (e.g., surgical settings) in the decision to order a pharmacogenetic test but typically turnaround times do not make or break a decision. The affordability of the test however, is one of the biggest concerns when ordering a test. Testing costs vary and are typically not covered by third party payers, although health systems, insurance companies, and employers are beginning to offer reimbursement mechanisms for pharmacogenetic testing. Until this is universal, the financial burden of pharmacogenetic testing will continue to sit with the patient, perpetuating access inequalities and creating missed opportunities to improve quality of care.

\section{CONCLUSION}

The increase in laboratories offering pharmacogenetic testing has created opportunities to advance safe and patient-tailored use of prescription drugs in unison with good clinical monitoring. However, the lack of regulation and standardization of these tests has made it challenging for providers to confidently utilize them. We are confident that these challenges will be overcome and that pharmacogenetic testing will become standard practice and ultimately be routinely integrated into healthcare systems. Until then, we encourage providers to consider the topics and consult the resources we have presented here, when selecting pharmacogenetic tests.

This article is protected by copyright. All rights reserved 


\section{ACKNOWLEDGEMENTS}

C.A.B. is supported by the Cumming School of Medicine and Alberta Children's Hospital Foundation.

\section{REFERENCES}

1. Haga SB, Kantor A. Horizon Scan Of Clinical Laboratories Offering Pharmacogenetic Testing. Health Aff (Millwood). 2018;37(5):717-723.

2. Pratt VM, Del Tredici AL, Hachad H, et al. Recommendations for Clinical CYP2C19 Genotyping Allele Selection: A Report of the Association for Molecular Pathology. J Mol Diagn. 2018;20(3):269-276.

3. Bousman CA, Dunlop BW. Genotype, phenotype, and medication recommendation agreement among commercial pharmacogenetic-based decision support tools.

Pharmacogenomics J. 2018;18(5):613-622.

4. PharmGKB. Gene-specific Information Tables for CYP2D6. Accessed February 12, 2019.

\section{FIGURE LEGENDS}

Figure 1. Decision tree for the selection of a clinical pharmacogenetic (PGX) test. CAP = College of American Pathologists; CLIA = Clinical Laboratory Improvement Amendments; CPIC = Clinical Pharmacogenetics Implementation Consortium; IGNITE = Implementing Genomics in Practice; PharmGKB = Pharmacogenomics Knowledgebase; PharmVar = Pharmacogene Variation Consortium.

Figure 2. Gene-drug pairs with pharmacogenetic-based guidelines published by the Clinical Pharmacogenetics Implementation Consortium, Royal Dutch Association for the Advancement of Pharmacy - Pharmacogenetics Working Group, or the Canadian Pharmacogenomics Network for Drug Safety. ADHD, attention-deficit/hyperactivity disorder; PPI, proton pump inhibitors.

This article is protected by copyright. All rights reserved 


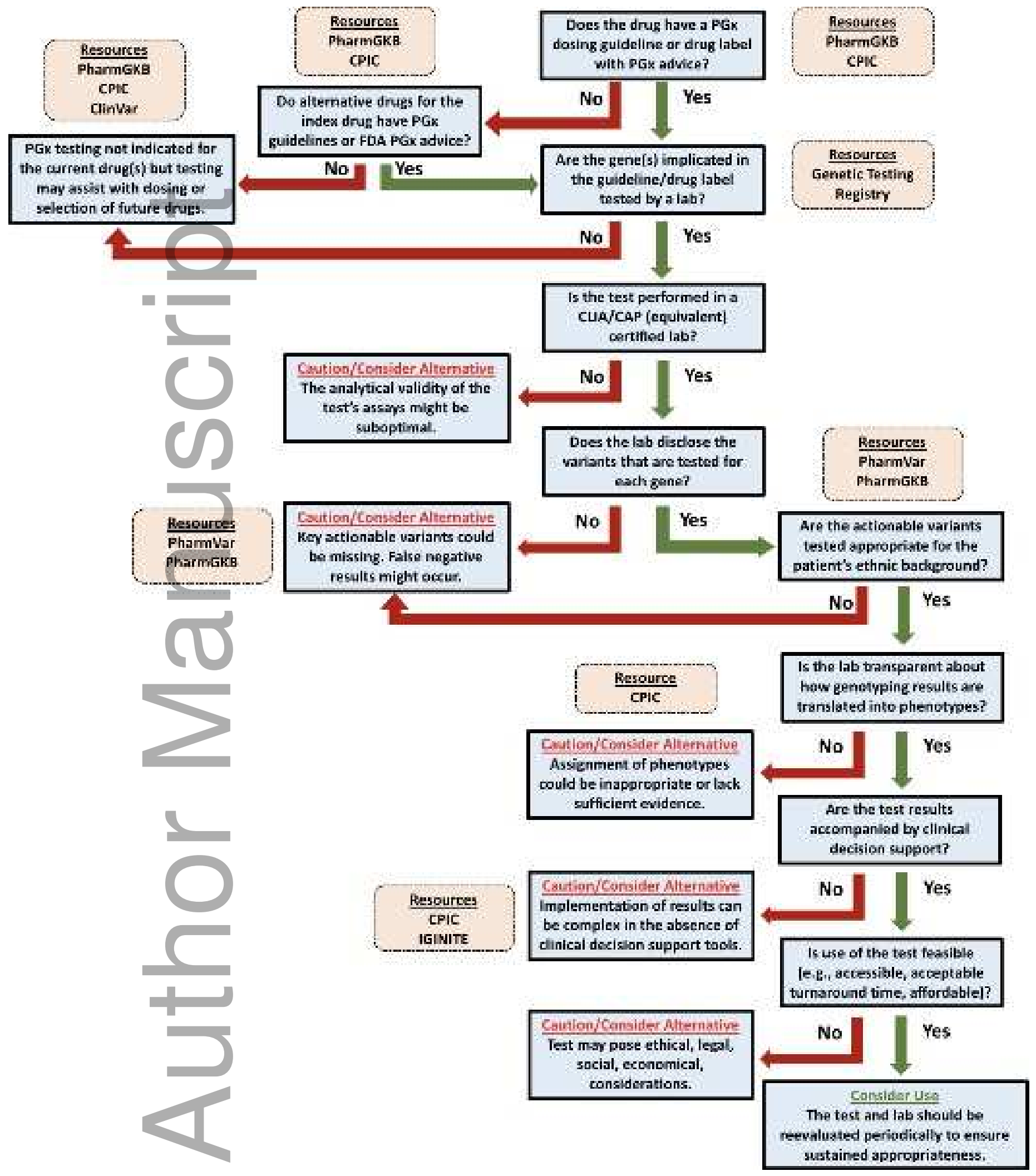

cpt_1432_f1.tif

This article is protected by copyright. All rights reserved 


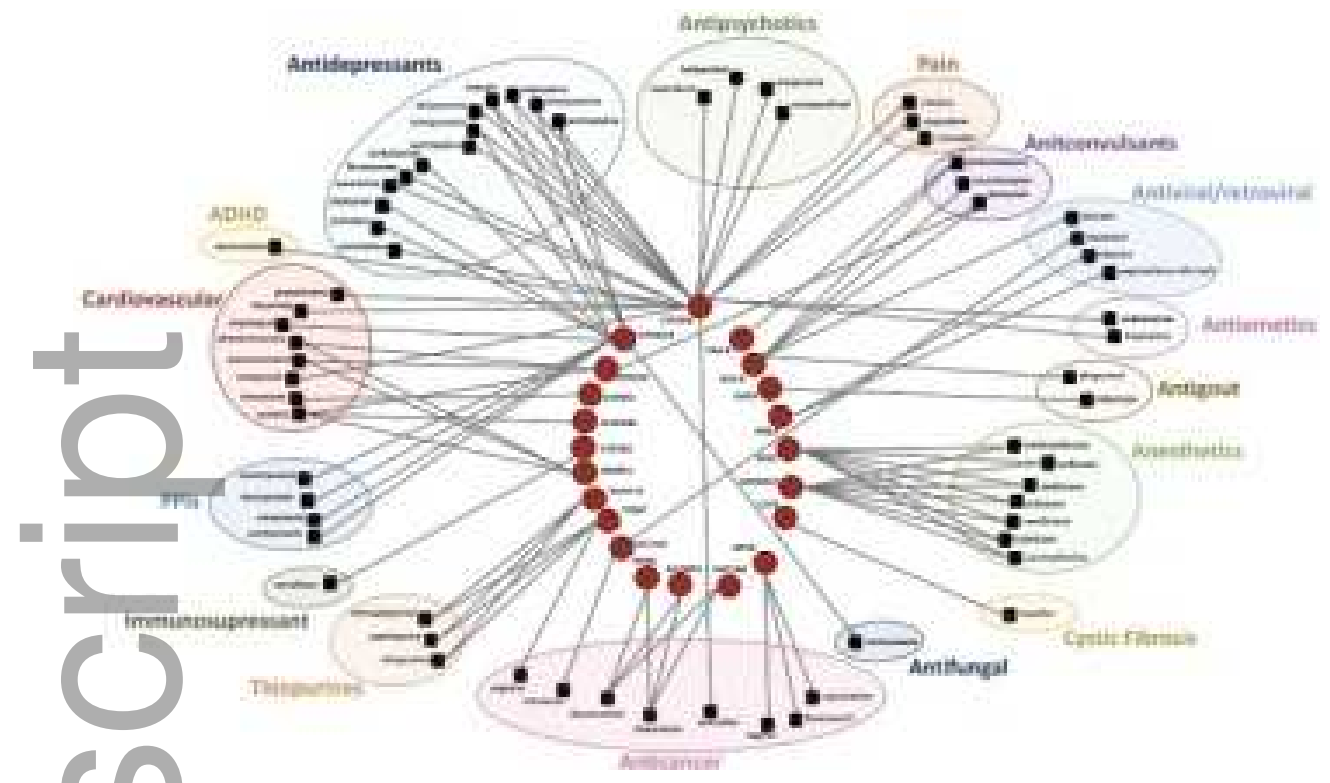

cpt_1432_f2.tif
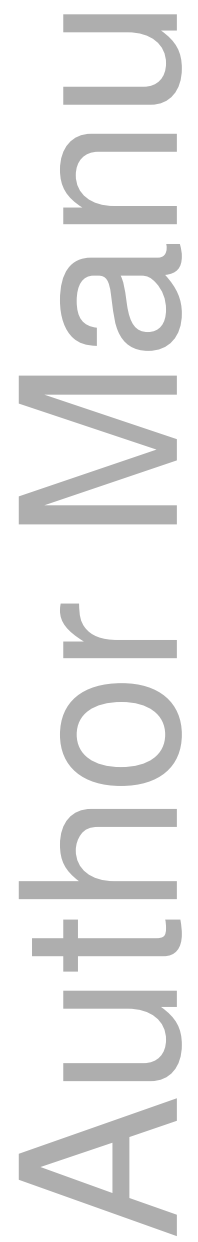

This article is protected by copyright. All rights reserved 


\section{University Library}

\section{- M M I E E R VA A gateway to Melbourne's research publications}

Minerva Access is the Institutional Repository of The University of Melbourne

Author/s:

Bousman, CA;Zierhut, H;Müller, DJ

Title:

Navigating the Labyrinth of Pharmacogenetic Testing: A Guide to Test Selection.

Date:

2019-08

Citation:

Bousman, C. A., Zierhut, H. \& Müller, D. J. (2019). Navigating the Labyrinth of

Pharmacogenetic Testing: A Guide to Test Selection.. Clin Pharmacol Ther, 106 (2),

pp.309-312. https://doi.org/10.1002/cpt.1432.

Persistent Link:

http://hdl.handle.net/11343/285705 